\title{
Kinder werden fürs Einnässen bestraft
}

Eine primäre nächtliche Enuresis betrifft bis zu 10\% aller Kinder im Alter von 7 Jahren. Klare Erkenntnisse zu Ätiologie und Risikofaktoren fehlen bisher ebenso wie einfache Therapiemaßnahmen. Häufig bestrafen Eltern ihre Kinder für das Einnässen, und die Dunkelziffer in diesem Zusammenhang dürfte erheblich sein. Brasilianische Mediziner haben untersucht, wie Eltern mit einnässenden Kindern umgehen und ob sie diese bestrafen. J Urol 2016; 195: 1227-1231

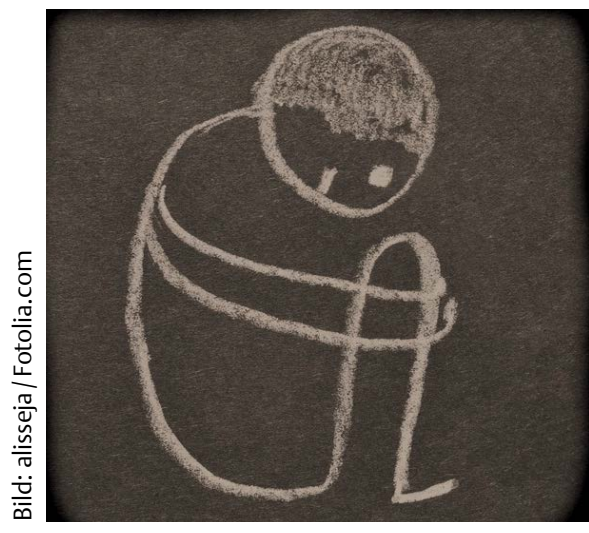

Kommentar

\section{Urotherapeutische Beratung für Eltern erforderlich!}

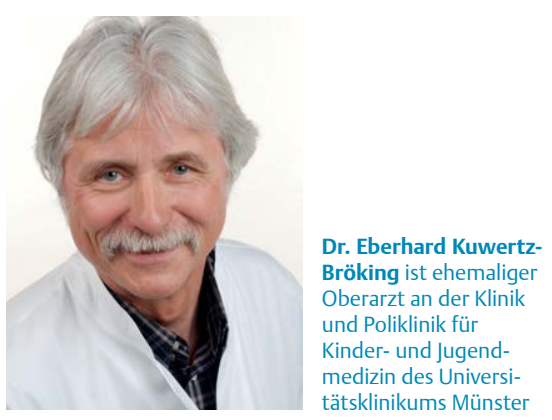

In der vorliegenden Studie aus Brasilien wird berichtet, dass fast alle Kinder mit Enuresis im untersuchten Kollektiv auf unterschiedliche Weise für das Einnässen bestraft werden; 41,4\% in dieser Studie erlitten körperliche Gewalt. Erstaunlicherweise wird Gewalt besonders häufig in Familien beobachtet, in denen bereits Eltern an einer Enuresis litten. Bestrafungen werden häufig von Müttern ausgeübt.

Die Bestrafung von Kindern für Einnässen ist in der beschriebenen Gruppe die Regel, nicht die Ausnahme, so die Autoren, und fast die Hälfte der Kinder wird nicht nur verbal, sondern auch mit Schlägen gestraft. Eltern sollten unbedingt darüber aufgeklärt werden, dass es sich bei der Enuresis nicht um ein absichtliches „schlechtes Benehmen“ ihrer Kinder handelt, sondern dass eine Vielzahl neurologischer, physiologischer und emotionaler Gründe dafür existieren kann, die nicht willkürlich kontrolliert und auf keinen Fall mit Strafen behoben werden können, sondern professionelle Betreuung benötigen, fassen die Autoren zusammen.

Dr. Elke Ruchalla, Bad Dürrheim
Es ist bekannt, dass Eltern, vor allem die Mütter, durch die Enuresis belastet sind. Studien zur Lebensqualität zeigen deutliche Hinweise auf eine erhebliche Beeinträchtigung [1, 2]. Egemen et al. [3] zeigten einen höheren Depressionsscore bei betroffenen Müttern und Naitoh et al. [4] berichteten, dass nicht nur die Lebensqualität vermindert, sondern auch ein höheres Maß an Ängstlichkeit (jedoch keine erhöhte Depressivität) nachweisbar war. Erfreulicherweise besserten sich beide Parameter nach erfolgreicher Behandlung.

Gut informierte Eltern unterstützen ihre Kinder häufig in positiver Weise bei der Behandlung einer Enuresis. Aber viele El-
Die Auswertung ergab, dass alle Kinder in der einen oder anderen Form für ihr nässen bestraft wurden. Dazu gehörten in hungen, Demütigungen und Beleidigungen), aber mehr als $40 \%$ der Kinder $(n=36)$ wurden auch geschlagen. 67 Eltern bzw. Elternteile hatten in ihrer Kindheit ebenfalls eingenässt, 57 von ihnen waren von ihren Eltern ebenfalls bestraft worden. $60 \%$ der Eltern hatten einen niedrigen Bildungsstand. 
tern reagieren auch heute noch auf das nächtliche Einnässen mit Tadeln, dem Verweigern von kindlichen Wünschen (z.B. Fernsehen), demütigenden Bemerkungen, aber auch harten Bestrafungen bis hin zu körperlicher Gewalt. Die Bandbreite elterlicher Gefühle schwankt zwischen Wut und Aggressivität auf der einen Seite und Schuldgefühlen und Selbstzweifeln auf der anderen Seite, verbunden mit dem Gefühl, schwerwiegende Erziehungsfehler gemacht zu haben. Ein ungünstiger Sozial- und Bildungsstatus, aber auch kulturelle Aspekte begünstigen elterliche Intoleranz. Manche Eltern sind davon überzeugt, dass Bestrafungen zur Behandlung des Einnässens durchaus sinnvoll sind.

In den aktuell empfohlenen und verwendeten standardisierten Anamnesefragebögen sind Screeningfragen zu möglicher psychologischer / psychiatrischer Komorbidität einer Harninkontinenz enthalten [5]. Reaktionen der Eltern auf das Einnässen und ihr Verhalten gegenüber den Kindern werden jedoch nicht detailliert erfragt. Üblich ist die Frage nach dem Leidensdruck, den Eltern und Kinder auf einer Skala bewerten können.

\section{Die Studie weist auf eine wichtige Problematik hin}

Dem Anamnesegespräch kommt daher eine besondere Bedeutung zu. Erfasst werden sollte, in welchem Ausmaß unangenehme Gefühle wie Scham oder Schuld mit dem Einnässproblem verknüpft sind und welche Vorstellungen die Eltern von „normaler“ Kontinenzentwicklung und von einem „Sauberkeitstraining“ haben. Bedeutsam ist die Frage an die Eltern, welche Vorstellung sie über die Ursache des Einnässens haben, ob eine „psychische Ursache“ angenommen wird oder ob eine Absicht des Kindes unterstellt wird. Milde Formen von Bestrafung werden häufig zugegeben, aber härtere Strafen bleiben meist ein Familiengeheimnis. Die vorliegende Arbeit weist eindrücklich auf diese Problematik hin.

Elterliche Unkenntnis und Hilflosigkeit führen zur Intoleranz gegenüber dem Problem. Die Autoren betonen daher $\mathrm{zu}$ Recht, dass Eltern im Umgang mit ihrem einnässenden Kind Hilfe benötigen.

Die aktuelle AWMF-Leitlinie empfiehlt zu Beginn einer Behandlung einer Enuresis urotherapeutische Maßnahmen [5]. Im Vordergrund der Beratung während und nach Abschluss der Diagnostik steht die Entlastung von Kind und Eltern und die Entwicklung einer gemeinsamen Strategie und Motivation für die weitere Behandlung.

Eltern sollten darüber informiert werden, dass es sich bei der Enuresis nur extrem selten um eine organische oder psychische Erkrankung handelt, sondern um eine Reifungsverzögerung der nächtlichen Blasenkontrolle, die einer bewussten Kontrolle nicht zugänglich ist („Information") und deren Behandlung Zeit und Geduld erfordert. Das Einnässen ist kein Symptom unzureichender oder falscher Erziehung und kein bewusster Akt des Kindes („Entmystifizierung“). Informationen zur normalen Blasenkontrolle am Tag und in der Nacht und Kenntnisse über die Ätiologie der Enuresis sollten verständlich vermittelt werden. Reaktionsmuster der Eltern, familiäre Konflikte und Bestrafungen in Zusammenhang mit der Enuresis sind, wenn möglich, anzusprechen.

Fazit

Eine ausreichende Kenntnis über ätiologische und therapeutische Aspekte einer Enuresis ist für die Unterstützung der Kinder, für elterliche Toleranz, Motivation, Vermeidung von Bestrafung und somit für den gewünschten Behandlungserfolg von großer Bedeutung.

\section{Dr. Eberhard Kuwertz-Bröking, Münster}

\section{Literatur}

1 Von Gontard A, Baeyens D, Van Hoecke E et al. Psychological and psychiatric issues in urinary and fecal incontinence. J Urol 2011; 185: 1432-1437

2 Kilicoglu AG, Mutlu C, Bahali MK et al. Impact of enuresis nocturna on health-related quality of life in children and their mothers. J Pediatr Urol 2014; 10: 1261-1266

3 Egemen A, Akil I, Canda E et al. An evaluation of quality of life of mothers of children with enuresis nocturna. Pediatr Nephrol 2008 23: 93-98

4 Naitoh Y, Kawauchi A, Soh J et al. Health related quality of life for monosymptomatic enuretic children and their mothers. J Urol 2012; 188: 1910-1914

5 Deutsche Gesellschaft für Kinder- und Jugendmedizin (DGKJ) und Deutsche Gesellschaft für Kinder- und Jugendpsychiatrie, Psychosomatik und Psychotherapie (DG$\mathrm{KJP}$ ). Enuresis und nicht-organische (funktionelle) Harninkontinenz bei Kindern und Jugendlichen. 2. 12. 2015. Im Internet: http://www.awmf.org/leitlinien/detail/ II/028-026.html 